\title{
Corrigendum: Classification and characterization of microsatellite instability across 18 cancer types
}

Ronald J Hause, Colin C Pritchard, Jay Shendure \& Stephen J Salipante

Nat. Med. 22, 1342-1350 (2016); published online 03 October 2016; corrected after print 19 July 2017; corrected after print 28 December 2017

In the version of this article initially published, the numbers on the $y$-axis of Figure $2 \mathrm{~b}$ were incorrect by a power of 10 , and the numbers in the text describing the frequency of MSI-H tumors were also incorrect. The original text read: "Still lower, but detectable, frequencies of MSI-H were observed in 12 other cancer types; collectively, one or more individual MSI-H tumors were identified in 16 of the 18 cancer types examined". This has been corrected to: "Still lower, but detectable, frequencies of MSI-H were observed in 10 other cancer types; collectively, one or more individual MSI-H tumors were identified in 14 of the 18 cancer types examined". These errors do not impact the findings or conclusions of this work; however, they have been corrected for accuracy. The error has been corrected in the HTML and PDF versions of the article.

Corrigendum: Suppression of luteinizing hormone enhances HSC recovery after hematopoietic injury

Enrico Velardi, Jennifer J Tsai, Stefan Radtke, Kirsten Cooper, Kimon V Argyropoulos, Shieh Jae-Hung, Lauren F Young, Amina Lazrak, Odette M Smith, Sophie Lieberman, Fabiana Kreines, Yusuke Shono, Tobias Wertheimer, Robert R Jenq, Alan M Hanash, Prema Narayan, Zhenmin Lei, Malcolm A Moore, Hans-Peter Kiem, Marcel R M van den Brink \& Jarrod A Dudakov

Nat. Med.; doi:10.1038/nm.4470; corrected online 22 January 2018

In the version of this article initially published online, the phrase "ex vitro" appears in the abstract. This should be "ex vivo". The error has been corrected in the print, PDF and HTML versions of this article.

\section{Corrigendum: Positively selected enhancer elements endow osteosarcoma cells with metastatic competence}

James J Morrow, Ian Bayles, Alister P W Funnell, Tyler E Miller, Alina Saiakhova, Michael M Lizardo, Cynthia F Bartels, Maaike Y Kapteijn, Stevephen Hung, Arnulfo Mendoza, Gursimran Dhillon, Daniel R Chee, Jay T Myers, Frederick Allen, Marco Gambarotti, Alberto Righi, Analisa DiFeo, Brian P Rubin, Alex Y Huang, Paul S Meltzer, Lee J Helman, Piero Picci, Henri H Versteeg, John A Stamatoyannopoulos, Chand Khanna \& Peter C Scacheri

Nat. Med. 24, 176-185 (2018); published online 15 January 2018; corrected after print 7 February 2018

In the version of this article initially published, two of the authors are incorrectly identified as John Stamatoyannopolus and Henri Versteeg. The authors' names are John A Stamatoyannopoulos and Henri H Versteeg. Also, the affiliation "Research Laboratory, Istituto Ortopedico Rizzoli, Bologna, Italy" is incorrect. The correct affiliation is "Laboratory of Experimental Oncology, Istituto Ortopedico Rizzoli, Bologna, Italy". The errors have been corrected in the HTML and PDF versions of the article.

Corrigendum: Sex-specific transcriptional signatures in human depression

Benoit Labonté, Olivia Engmann, Immanuel Purushothaman, Caroline Menard, Junshi Wang, Chunfeng Tan, Joseph R Scarpa, Gregory Moy, Yong-Hwee E Loh, Michael Cahill, Zachary S Lorsch, Peter J Hamilton, Erin S Calipari, Georgia E Hodes, Orna Issler, Hope Kronman, Madeline Pfau, Aleksandar L J Obradovic, Yan Dong, Rachael L Neve, Scott Russo, Andrew Kasarskis, Carol Tamminga, Naguib Mechawar, Gustavo Turecki, Bin Zhang, Li Shen \& Eric J Nestler

Nat. Med. 23, 1102-1111 (2017); published online 21 August 2017; corrected after print 7 March 2018

In the version of this article initially published, the last name of author Andrew Kasarskis was misspelled as "Kazarskis". The error has been corrected in the PDF and HTML versions of the article.

Corrigendum: Genetic identification of thiosulfate sulfurtransferase as an adipocyte-expressed antidiabetic target in mice selected for leanness

Nicholas M Morton, Jasmina Beltram, Roderick N Carter, Zoi Michailidou, Gregor Gorjanc, Clare McFadden, Martin E Barrios-Llerena, Sergio Rodriguez-Cuenca, Matthew T G Gibbins, Rhona E Aird, José Maria Moreno-Navarrete, Steven C Munger, Karen L Svenson, Annalisa Gastaldello, Lynne Ramage, Gregorio Naredo, Maximilian Zeyda, Zhao V Wang, Alexander F Howie, Aila Saari, Petra Sipilä, Thomas M Stulnig, Vilmundur Gudnason, Christopher J Kenyon, Jonathan R Seckl, Brian R Walker, Scott P Webster, Donald R Dunbar, Gary A Churchill, Antonio Vidal-Puig, José Manuel Fernandez-Real, Valur Emilsson \& Simon Horvat Nat. Med. 22, 771-779 (2016); published online 6 June 2016; corrected after print 7 March 2018 